\title{
Analysis of the correlation between plasma coagulation factor VII, PAI-1, and uric acid with insulin resistance and macrovascular complications in elderly patients with type 2 diabetes
}

\author{
Lei $\mathrm{Li}^{1}$, Zhenfeng Shi ${ }^{1}$, Lisha $\mathrm{Ma}^{2}$, Yi Lu${ }^{1}$ \\ ${ }^{1}$ Department of Endocrinology, Xingtai People's Hospital, Xingtai, China; ${ }^{2}$ Pediatric Department, The Eighth People's Hospital of Qingdao, \\ Qingdao, China \\ Contributions: (I) Conception and design: L Li; (II) Administrative support: L Li; (III) Provision of study materials or patients: Z Shi, L Ma; (IV) \\ Collection and assembly of data: Z Shi, L Ma; (V) Data analysis and interpretation: Y Lu; (VI) Manuscript writing: All authors; (VII) Final approval \\ of manuscript: All authors. \\ Correspondence to: Lei Li. No. 16, Hongxing Street, Xingtai, China. Email: lilei2020811@126.com.
}

Background: To analyze the correlations between plasma coagulation factor VII (FVII), plasma plasminogen activator inhibitor-1 (PAI-1), uric acid, and insulin resistance (IR) and diabetic macroangiopathy (DMAP) in elderly patients with type 2 diabetes mellitus (T2DM).

Methods: The clinical data of 112 elderly T2DM patients admitted to our hospital between March 2017 and March 2020 were enrolled. Subgroups were allocated based on the inclusion and exclusion criteria, body mass index (BMI), homeostasis model assessment (HOMA)-IR and the presence of DMAP. The patients were allocated as the study objects (T2DM group, $n=90$ ), simple obesity (BMI $\geq 25 \mathrm{~kg} / \mathrm{m}^{2}, \mathrm{n}=25$ ), simple nonobese (BMI $<25 \mathrm{~kg} / \mathrm{m}^{2}, \mathrm{n}=22$ ), obesity with DMAP ( $\left.\mathrm{n}=24\right)$, and non-obese with DMAP ( $\left.\mathrm{n}=19\right)$. Meanwhile, data from 50 healthy controls from physical examinations were employed. The levels of FVII, PAI-1, HOMA-IR and uric acid were determined. Pearson correlation analysis was used to measure the correlations, and the receiver operating characteristic (ROC) curve was used to determine the predictive value of detection indexes.

Results: The levels of FVII, PAI-1, and uric acid in the T2DM group were higher than those of the control group $(\mathrm{P}<0.05)$. In terms of the levels of FVII, PAI-1, and uric acid: simple non-obese group < simple obese group < non-obese with DMAP group < obese with DMAP group $(\mathrm{P}<0.05)$. The levels of FVII, PAI1 , and uric acid in T2DM patients were positively correlated with $\mathrm{BMI}$ and Homeostasis model assessment (HOMA)-IR $(\mathrm{P}<0.05)$. The ROC curve results showed that the area under the curve (AUC) for FVII, PAI-1, and uric acid separately, as well as a combination of the three were $0.930,0.831,0.888$, and 0.997 , respectively.

Conclusions: FVII, PAI-1, and uric acid levels are elevated in elderly T2DM patients. The levels of FVII, PAI-1, and uric acid are closely related to IR in elderly T2DM patients. The combination of TFVII, PAI1 , and uric acid levels can be used as an effective measure to predict patients with DMAP. Furthermore, the levels of these markers should be promptly measured in a clinical setting for early intervention and improvement of the patient's outcomes.

Keywords: Type 2 diabetes (T2DM); plasma coagulation factor VII (plasma coagulation FVII); plasminogen activator inhibitor-1 (PAI-1); uric acid; insulin resistance (IR); macroangiopathy

Submitted Dec 03, 2020. Accepted for publication Jan 19, 2021.

doi: 10.21037/apm-20-2609

View this article at: http://dx.doi.org/10.21037/apm-20-2609 


\section{Introduction}

Diabetes (diabetes mellitus, DM) is a complex metabolic disorder characterized by hyperglycemia due to insulin (INS) secretion defects or impaired biological response. Type 2 diabetes mellitus (T2DM) is caused by insufficient secretion of INS by pancreatic $\beta$ cells or an insensitivity of target cells to INS, also known as INS-independent diabetes (1). Studies have shown that diabetic macroangiopathy (DMAP) is the main cause of disability and death in T2DM diabetes patients, and insulin resistance (IR) is one of the risk factors for DMAP (2). Therefore, early intervention in the IR population can prevent or delay the occurrence and development of T2DM and DMAP.

Recently, studies have suggested that the hypercoagulable state of blood is associated with the occurrence of IR, which includes plasma coagulation factor VII (FVII) and plasma plasminogen activator inhibitor-1 (PAI-1) (3). Also, clinical practice has found that elevated basal uric acid levels are associated with the occurrence and mortality of cardiovascular diseases (4). In this study, by observing the different FVII, PAI-1, and uric acid levels in elderly T2DM patients and investigating their IR and DMAP status, we aimed to explore the associations between FVII, PAI-1, uric acid and IR and DMAP in elderly T2DM patients, as well as examining the roles of these markers in the occurrence of DMAP.

We present the following article in accordance with the STARD reporting checklist (available at http://dx.doi. org/10.21037/apm-20-2609).

\section{Methods}

\section{General information}

The clinical data of 112 elderly T2DM patients admitted to our hospital between March 2017 and March 2020 were selected. The inclusion criteria were as follows: (I) patients meeting the diagnostic criteria for T2DM (5); (II) patients over 60 years of age; and (III) patients with complete clinical data. Patients were excluded based on the following criteria: (I) those with type 1 diabetes, a special type of diabetes, or acute diabetes complications; (II) those with acute and chronic infections, tumors, blood system diseases, cardiovascular and cerebrovascular diseases, or liver and kidney dysfunction; and (III) those with rheumatic diseases or other endocrine and metabolic diseases, such as abnormal pituitary function, abnormal thyroid function, abnormal adrenal cortex function, etc. Based on the aforementioned inclusion and exclusion criteria, a total of 90 patients were selected as the study objects (T2DM group). Of these patients, 43 were males and 47 were females (aged 60-80 years, with an average age of $72.41 \pm 5.73$ years). According to the body mass index (BMI) and the presence or absence of DMAP, the patients were divided into a simple obesity group (BMI $\geq 25 \mathrm{~kg} / \mathrm{m}^{2}$, $\mathrm{n}=25$ ), a simple non-obese group (BMI $<25 \mathrm{~kg} / \mathrm{m}^{2}, \mathrm{n}=22$ ), an obesity with DMAP group $(\mathrm{n}=24)$, and a non-obese with DMAP group $(n=19)$. There were no significant differences in the general data of the four groups and they were comparable $(\mathrm{P}>0.05)$.

At the same time, 50 patients with normal physical examination results in our hospital during the same period were selected as the control group. The control group had complete clinical data. There were 24 males and 26 females ranging from 60 to 79 years of age, with an average age of $71.93 \pm 5.74$ years. There were no notable difference in the general information between the control group and the T2DM group, and they were comparable $(\mathrm{P}>0.05)$.

All procedures performed in this study involving human participants were in accordance with the Declaration of Helsinki (as revised in 2013). The study was approved by the Ethics Committee of Xingtai People's Hospital and written informed consent was obtained from all patients.

\section{Research methods}

Clinical data of all study subjects were collected, and the levels of FVII, PAI-1, and uric acid were compared between the control group and the T2DM group. The levels of FVII, PAI-1, and uric acid in the four subgroups of T2DM patients were also analyzed, and Pearson correlation analysis was used to measure the correlations between FVII, PAI-1, uric acid levels, and IR. The receiver operating characteristic (ROC) curve was used to determine the predictive values of FVII, PAI-1, and uric acid levels for patients with T2DM complicated with DMAP.

\section{Observation indicators}

\section{Level of FVII}

Three $\mathrm{mL}$ of fasting venous blood from was collected from all subjects. The blood was anticoagulated with $3.2 \%$ sodium citrate $(9: 1)$, centrifuged (1,000 r/min, $10 \mathrm{~min})$, and the plasma was subsequently extracted. For the plasma extraction, all of the specimens were quickly reconstituted in a $37^{\circ} \mathrm{C}$ water bath before the experiment. Reagent preparation: $1 \mathrm{~mL}$ of distilled water was added to each 
Table 1 Comparison of FVII, PAI-1, and uric acid levels between the T2DM and control groups $(\bar{x} \pm s)$

\begin{tabular}{lcccc}
\hline Groups & Patients & FVII $(\%)$ & PAl-1 $(\mathrm{ng} / \mathrm{mL})$ & Uric acid $(\mu \mathrm{mol} / \mathrm{L})$ \\
\hline T2DM group & 90 & $131.21 \pm 21.25$ & $82.26 \pm 20.41$ & $546.16 \pm 66.26$ \\
Control group & 50 & $103.58 \pm 20.46$ & $63.25 \pm 19.35$ & $226.45 \pm 51.21$ \\
$\mathrm{t}$ & - & 7.469 & 5.378 & 44.696 \\
$\mathrm{P}$ & - & $<0.001$ & $<0.001$ & $<0.001$ \\
\hline
\end{tabular}

FVII, factor VII; PAI-1, plasminogen activator inhibitor-1; T2DM, type 2 diabetes mellitus.

bottle to reconstitute, and it was kept at room temperature for 30 minutes and mixed gently to make it a homogeneous suspension. After calibration, quality control, and debugging of the STA series analyzer (Stago, France), the specimens were tested. The test was carried out according to the manufacturer's instructions, and the samples were automatically measured by the STA analyzer using the magnetic bead method.

\section{Level of PAI-1}

Three $\mathrm{mL}$ of fasting venous blood was collected from all subjects, anticoagulated with $3.2 \%$ sodium citrate $(9: 1)$, and centrifuged $(3,000 \mathrm{r} / \mathrm{min}, 10 \mathrm{~min})$. The plasma was extracted and stored below $-20{ }^{\circ} \mathrm{C}$. The same batch of measurement was carried out using the enzyme-linked immunosorbent double antibody sandwich method, and the kit was purchased from the Shanghai Sun Biotechnology Company (Shanghai, China).

\section{Uric acid level}

Three $\mathrm{mL}$ of fasting venous blood was collected from all subjects, and a Hitachi 7600 automatic biochemical analyzer (Japan) was used to measure the uric acid level.

\section{IR-related indicators}

Five $\mathrm{mL}$ of fasting venous blood was collected from all subjects. The Siemens automatic biochemical analyzer (Siemens, Japan) was used for detection. Fasting blood glucose (FBG) and fasting plasma insulin (FINS) were recorded in each group, and at the same time, the IR index at homeostasis was calculated using the homeostasis model assessment (HOMA). HOMA-IR is calculated by the: HOMA-IR= FPG $\times$ FINS/22.5 (6).

\section{Statistical methods}

The data in this study were statistically analyzed using
SPSS18.0 software (SPSS, USA). The count data were expressed as $\mathrm{n}(\%)$ using the chi square $\left(\chi^{2}\right)$ test, the measurement data were described with the mean \pm standard deviation $(\bar{x} \pm s)$, and the $\mathrm{F} / \mathrm{t}$ test was performed. Pearson correlation was used to analyze the correlation between FVII, PAI-1, uric acid levels and IR, and the ROC curve was used to analyze the predictive value of FVII, PAI-1, and uric acid levels in T2DM patients with DMAP. $\alpha=0.05$ was used to indicate a statistically significant difference.

\section{Results}

Comparison of FVII, PAI-1, and uric acid levels between the T2DM and control groups

The levels of FVII, PAI-1, and uric acid in the T2DM group were higher than those in the control group $(\mathrm{P}<0.05)$ (Table 1).

\section{Comparison of FVII, PAI-1, and uric acid levels in four subgroups}

Comparison of FVII, PAI-1, and uric acid levels: simple non-obese group $<$ simple obese group $<$ non-obese with DMAP group < obese with DMAP group $(\mathrm{P}<0.05)$ (Table 2$)$.

\section{The relationship between the FVII level and BMI and HOMA-IR in T2DM patients}

The FVII level in T2DM patients was positively correlated with BMI and HOMA-IR $(\mathrm{P}<0.05)$ (Table 3, Figure 1A,B).

\section{The relationship between PAI-1 level and BMI and HOMA-IR in T2DM patients}

The PAI-1 level in T2DM patients was positively correlated with BMI and HOMA-IR $(\mathrm{P}<0.05)$ (Table 4, Figure $2 A, B)$. 
Table 2 Comparison of FVII, PAI-1, and uric acid levels in the four subgroups $(\bar{x} \pm s)$

\begin{tabular}{lcccc}
\hline Groups & Patients & FVII $(\%)$ & PAl-1 $(\mathrm{ng} / \mathrm{mL})$ & Uric acid $(\mu \mathrm{mol} / \mathrm{L})$ \\
\hline Simple non-obese group & 25 & $112.26 \pm 20.56$ & $72.59 \pm 19.13$ & $517.59 \pm 65.37$ \\
Simple obese group & 22 & $124.25 \pm 20.43^{\mathrm{a}}$ & $80.93 \pm 19.86^{\mathrm{a}}$ & $543.97 \pm 65.28^{\mathrm{a}}$ \\
Non-obese with DMAP group & 19 & $135.14 \pm 21.19^{\mathrm{ab}}$ & $88.49 \pm 20.57^{\mathrm{ab}}$ & $576.63 \pm 65.14^{\mathrm{ab}}$ \\
Obese with DMAP group & 24 & $143.69 \pm 22.46^{\mathrm{abc}}$ & $98.67 \pm 20.43^{\mathrm{abc}}$ & $591.26 \pm 69.64^{\mathrm{abc}}$ \\
F & - & 9.93 & 7.47 & 5.88 \\
P & - & $<0.001$ & $<0.001$ & $<0.001$ \\
\hline
\end{tabular}

${ }^{a}$ : compared with the simple non-obese group, $\mathrm{P}<0.05{ }^{b}{ }^{\text {: }}$ compared with the simple obesity group, $\mathrm{P}<0.05$; ${ }^{c}$ : compared with the nonobese group with DMAP, $\mathrm{P}<0.05$. FVII, factor VII; PAI-1, plasminogen activator inhibitor-1; DMAP, diabetic macroangiopathy.

Table 3 The relationship between FVII level and BMI and HOMA-IR in T2DM patients

\begin{tabular}{lcc}
\hline \multirow{2}{*}{ Independent variable } & \multicolumn{2}{c}{ FVII } \\
\cline { 2 - 3 } & $\mathrm{r}$ & $\mathrm{P}$ \\
\hline BMI & 0.761 & 0.005 \\
HOMA-IR & 0.579 & 0.018 \\
\hline
\end{tabular}

FVII, factor VII; BMI, body mass index; T2DM, type 2 diabetes mellitus.

\section{The relationship between uric acid level and BMI and HOMA-IR in T2DM patients}

The level of uric acid in T2DM patients was positively correlated with BMI and HOMA-IR $(\mathrm{P}<0.05)$ (Table 5, Figure $3 A, B)$.

The predictive value of FVII, PAI-1, and uric acid levels for patients with T2DM complicated by DMAP

The ROC curve was used to determine the predictive value of TFVII, PAI-1, and uric acid levels for T2DM patients with DMAP. The area under the curve (AUC) for FVII, PAI-1, and uric acid, as well as the combination of the three was $0.930,0.831,0.888$, and 0.997 , respectively. The area was largest when three markers were used together (Table 6, Figure 4).

\section{Discussion}

$\mathrm{DM}$ is a syndrome caused by multiple factors and its pathogenesis is highly complex (7). The macrovascular and microvascular complications are prevalent, which leads

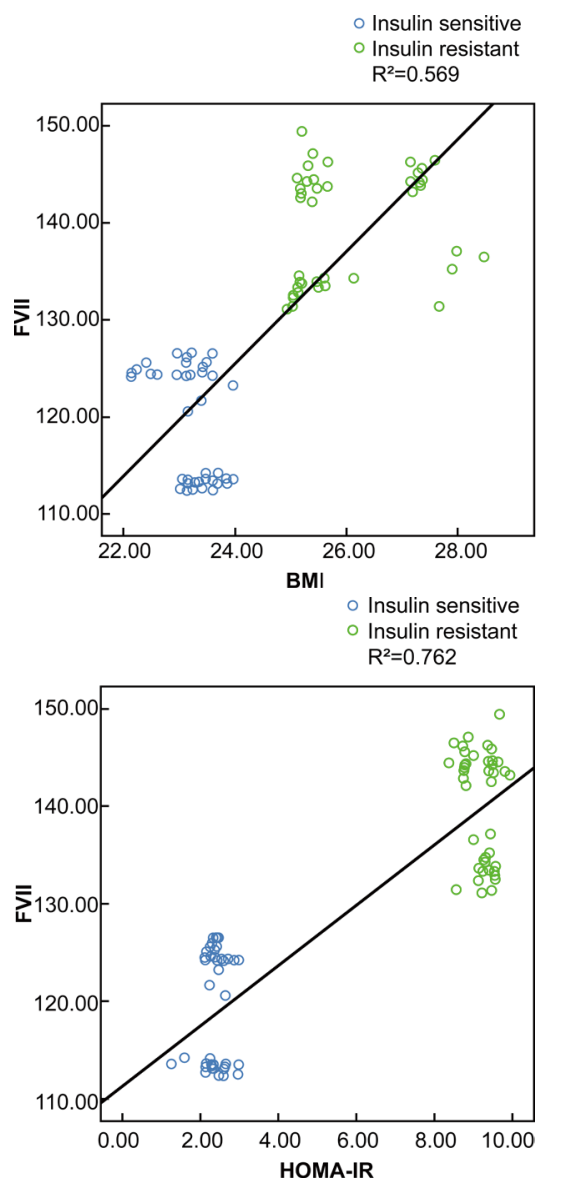

Figure 1 The relationship between FVII level and BMI and HOMA-IR in T2DM patients. FVII, factor VII; BMI, body mass index; T2DM, type 2 diabetes mellitus.

to a series of malignant complications including coronary heart disease, cardiomyopathy, arrhythmia, sudden death, and cerebrovascular disease (8). T2DM patients also have 
Table 4 The relationship between PAI-1 level and BMI and HOMA-IR in T2DM patients

\begin{tabular}{lcc}
\hline \multirow{2}{*}{ Independent variable } & \multicolumn{2}{c}{ PAl-1 } \\
\cline { 2 - 3 } & $\mathrm{r}$ & $\mathrm{P}$ \\
\hline BMI & 0.895 & 0.009 \\
HOMA-IR & 0.599 & 0.017 \\
\hline
\end{tabular}

PAl-1, plasminogen activator inhibitor-1; BMI, body mass index; T2DM, type 2 diabetes mellitus.

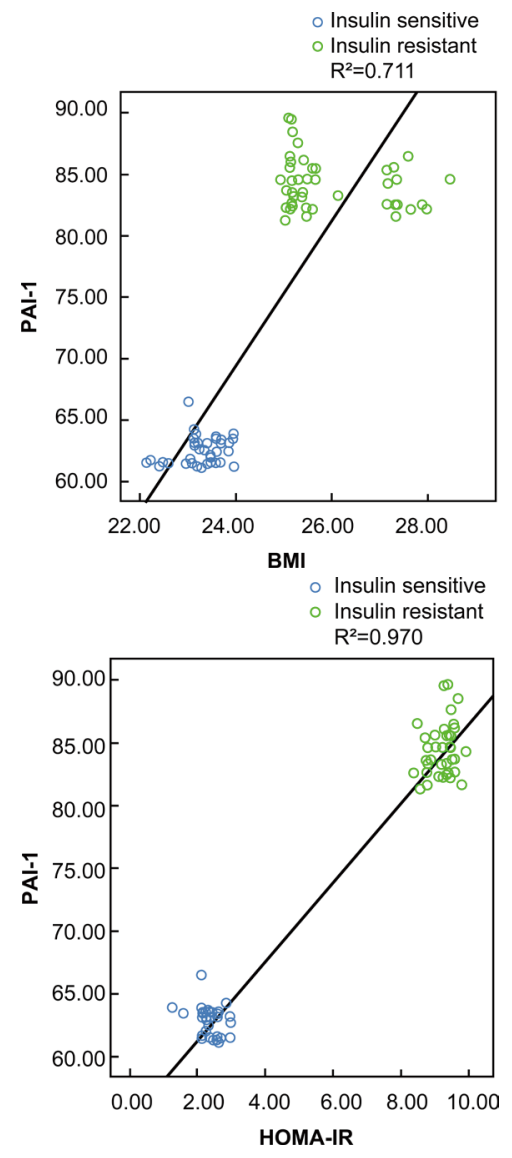

Figure 2 The relationship between PAI-1 level and BMI and HOMA-IR in T2DM patients. PAI-1, plasminogen activator inhibitor-1; BMI, body mass index; T2DM, type 2 diabetes mellitus.

increased risks of surgical complications, poor wound healing, and respiratory infection. Cardiovascular disease is the major cause of death in T2DM patients (9). Bariatric surgery is one of the most effective intervention to reduce mortality and cardiovascular events in the long run, as the risk for cardiovascular events was most significantly
Table 5 The relationship between uric acid level and BMI and HOMA-IR in T2DM patients

\begin{tabular}{lcc}
\hline \multirow{2}{*}{ Independent variable } & \multicolumn{2}{c}{ Uric acid } \\
\cline { 2 - 3 } & $\mathrm{r}$ & $\mathrm{P}$ \\
\hline BMI & 0.591 & 0.029 \\
HOMA-IR & 0.794 & 0.005 \\
\hline
\end{tabular}

BMI, body mass index; T2DM, type 2 diabetes mellitus.

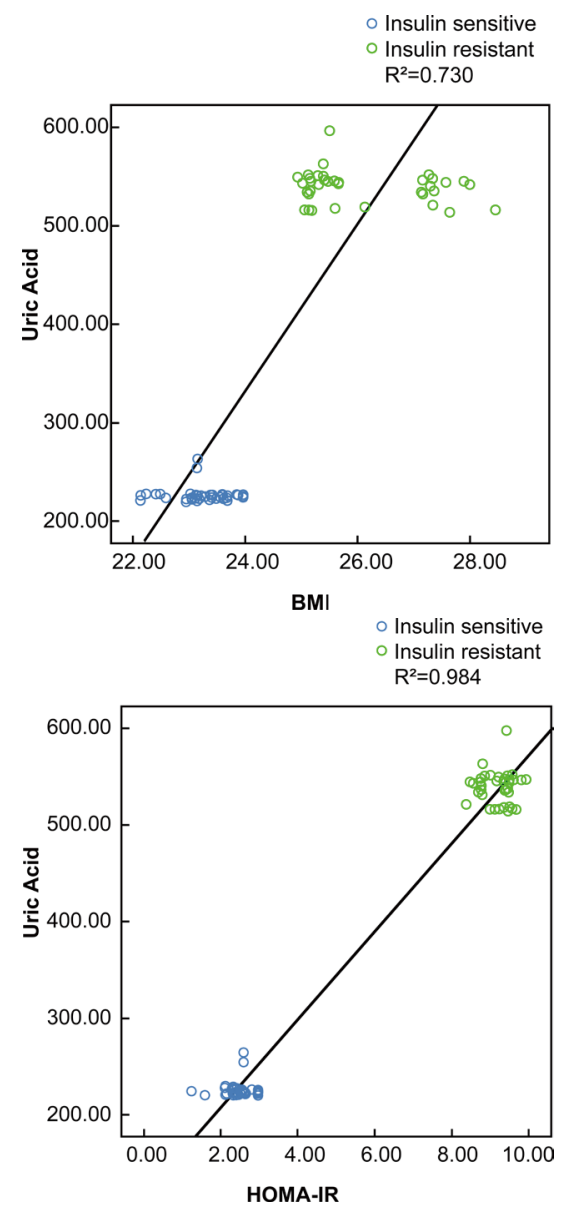

Figure 3 The relationship between uric acid level and BMI and HOMA-IR in T2DM patients. BMI, body mass index; T2DM, type 2 diabetes mellitus.

reduced (10). With the improvement of living standards, the incidence of T2DM has increased recently, and the related IR and DMAP have also attracted clinical attention.

Diabetic vascular disease is the main complication for DM patients; it is a key cause of death and disability that seriously threatens the life and health of patients (11). The 
Table 6 The predictive value of FVII, PAI-1, and uric acid levels in T2DM patients with DMAP

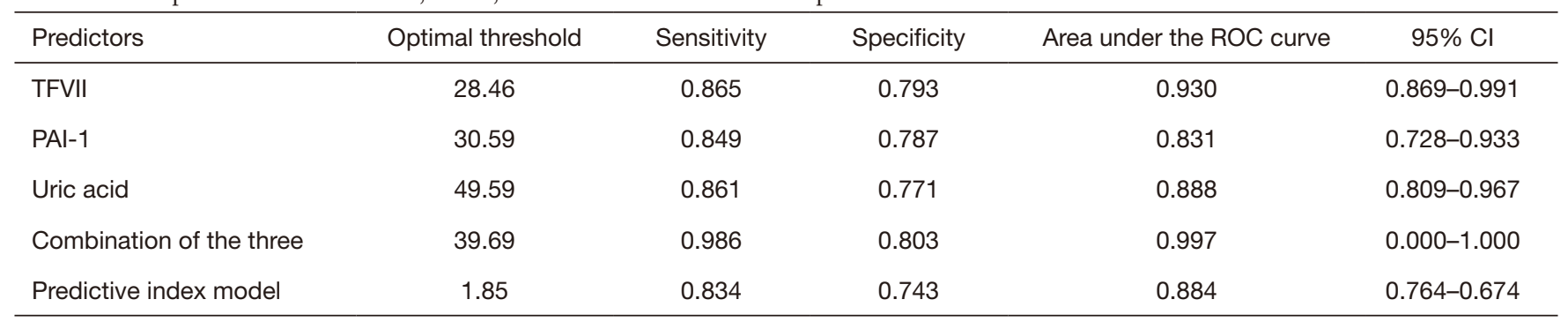

FVII, factor VII; PAI-1, plasminogen activator inhibitor-1; T2DM, type 2 diabetes mellitus; DMAP, diabetic macroangiopathy.

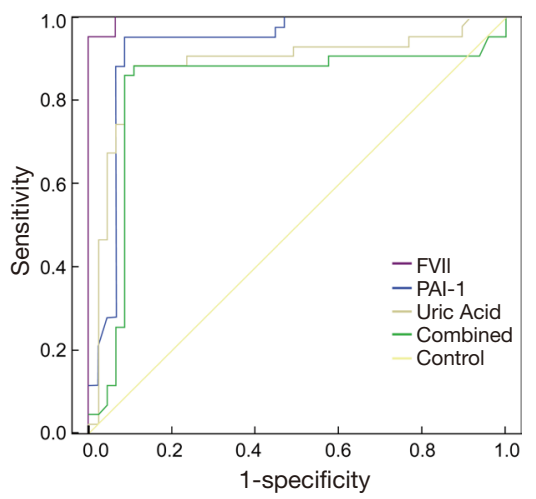

Figure 4 The predictive value of FVII, PAI-1, and uric acid levels for T2DM patients with DMAP. FVII, factor VII; PAI-1, plasminogen activator inhibitor-1; T2DM, type 2 diabetes mellitus; DMAP, diabetic macroangiopathy.

pathological basis of DMAP is atherosclerosis. Previous studies have shown that atherosclerosis is related to the following two factors: (I) hemorheological changes and abnormal blood viscosity can induce vascular endothelial cell damage and proliferation, lipid deposition, as well as series changes such as basement membrane thickening and blood coagulation, which can lead to decreased fibrinolysis and thrombus formation. Thrombotic factors can form atheromatous plaques on the surface of the blood vessel lumen, which ultimately leads to vascular occlusion (12). (II) Platelets can release procoagulant substances to activate thrombin, and thus, the blood is in a state of high viscosity, high coagulation, and high aggregation, which can result in microcirculation disorders, blood vessel self-ischemia, as well as hypoxic damage-triggered DMAP $(13,14)$.

IR refers to the biological effect of reduced binding of a certain amount of insulin to its specific receptor, and therefore, its corresponding effect cannot be exerted (15). IR syndrome includes a higher level of blood sugar, dyslipidemia, hyperinsulinemia, obesity, and other diseases, and studies have pointed out that blood hypercoagulability is also involved in the occurrence of IR (16). T2DM is a typical manifestation of IR. The loss of pancreatic $\beta$-cell secretory function to IR decompensation is a necessary condition for the pathogenesis of T2DM. HOMA-IR is a simple indicator to evaluate the body's IR, and it has a good correlation with the precise insulin sensitivity index measured by the hyperinsulinemic-positive grape clamp test. Moreover, a substantial body of evidence has shown that obesity is the main cause of IR (17). BMI is a risk factor that independently increases the prevalence of diabetes and can reflect the metabolic abnormalities of abdominal obesity. Therefore, this study used HOMA-IR and BMI to explore the relationship between the levels of FVII, PAI-1, uric acid, and IR in T2DM patients.

FVII is an important factor for the activation of the coagulation system. The activated FVII has a significant amplification effect on the coagulation process and can promote the formation of thrombi, and therefore, the detection of FVII can reflect whether the patient is in a hypercoagulable state (18). The results of this study showed that the level of FVII was higher in patients with T2DM, and was particularly obvious in patients with DMAP and/ or obesity, suggesting that FVII can participate in the occurrence and development of vascular disease and is closely related to T2DM and related complications.

In addition, the regulation of fibrinolytic activity in plasma also plays a key role in thrombosis. PAI-1 is the main inhibitor of the plasma fibrinolytic system. It can bind to the primary activators of the fibrinolytic system [plasminogen activator (tPA) and urokinase] and inhibit their activity, resulting in decreased fibrinolytic activity (19). Studies have shown that elevated plasma PAI-1 levels are a risk factor for atherosclerosis. This study found that PAI1 levels in patients with T2DM were significantly higher 
than those in the normal population $(\mathrm{P}<0.05)$, and are increased significantly in obese and DMAP patients. This indicates that PAI-1 may be a risk factor for T2DM patients with DMAP and metabolic syndrome. Furthermore, using Pearson correlation analysis, the results of this study showed that PAI-1 is positively correlated with BMI and HOMAIR, which is consistent with previous studies (20), and also strongly supports the interconnection between PAI1, obesity, IR, and atherosclerosis. A possible explanation is that, under IR, increased insulin and proinsulin in turn increase the synthesis of PAI-1 by endothelial and liver cells.

Serum uric acid is a metabolite of purine nucleoside. Studies have shown that patients with DM combined with hyperuricemia have a high tendency to IR. The possible mechanism is as follows: (I) obese people increase their energy intake and purine synthesis, which increases the level of uric acid; (II) patients with high BMI are often accompanied by abnormal lipid metabolism. Increased visceral fat increases free fatty acids in the portal system. When fatty acid synthesis in the liver increases, it can increase the production of triglycerides and uric acid; (III) in obesity, IR is caused by excessive free fatty acids that damage the insulin receptor system. High insulin levels can significantly promote urate reabsorption transporters and increase uric acid reabsorption; and (IV) IR can damage oxidative phosphorylation and increase the production of adenosine. The increased adenosine has the effect of retaining sodium and water, thereby increasing the reabsorption of uric acid (21). In recent years, uric acid has also received widespread attention as a risk factor for atherosclerosis. It is currently believed that the main pathophysiological mechanism involved in atherosclerosis is the formation of uric acid-platelet adhesion-oxygen free radicals and damage to coronary endothelial cells (22). The results of this study showed that uric acid levels in T2DM patients with obesity and/or DMAP were significantly increased. Pearson correlation analysis showed that uric acid levels were significantly correlated with BMI and HOMAIR in T2DM patients, suggesting that uric acid in T2DM patients was correlated with IR and DMAP. Therefore, controlling the level of uric acid in T2DM patients is expected to be an effective measure to reduce the impact of complications.

The ROC curve was further used to determine the predictive value of FVII, PAI-1, and uric acid levels in T2DM patients with DMAP. The results showed that the AUC was largest when the three measurements were combined, indicating that combined three-index detection can improve the predictive value of DMAP in T2DM patients.

In summary, the levels of FVII, PAI-1, and uric acid are higher in elderly T2DM patients, and the levels of TFVII, PAI-1, and uric acid are closely related to IR in elderly T2DM patients. The combination of TFVII, PAI-1, and uric acid levels can be used as an effective means to predict patients with DMAP. These levels should be detected clinically, and timely intervention is required to improve the prognosis of patients. The limitations of this study were that only a small number of cases were included, and the follow-up time was short, which may have an impact on the results of the study. In future, the sample size and followup time can be increased to further explore the relationship between FVII, PAI-1, uric acid levels and patient prognosis.

\section{Acknowledgments}

Funding: None.

\section{Footnote}

Reporting Checklist: The authors have completed the STARD reporting checklist. Available at http://dx.doi.org/10.21037/ apm-20-2609

Data Sharing Statement: Available at http://dx.doi. org/10.21037/apm-20-2609

Conflicts of Interest: All authors have completed the ICMJE uniform disclosure form (available at http://dx.doi. org/10.21037/apm-20-2609). The authors have no conflicts of interest to declare.

Ethical Statement: The authors are accountable for all aspects of the work in ensuring that questions related to the accuracy or integrity of any part of the work are appropriately investigated and resolved. All procedures performed in this study involving human participants were in accordance with the Declaration of Helsinki (as revised in 2013). The study was approved by the Ethics Committee of Xingtai People's Hospital and written informed consent was obtained from all patients.

Open Access Statement: This is an Open Access article distributed in accordance with the Creative Commons Attribution-NonCommercial-NoDerivs 4.0 International License (CC BY-NC-ND 4.0), which permits the non- 
commercial replication and distribution of the article with the strict proviso that no changes or edits are made and the original work is properly cited (including links to both the formal publication through the relevant DOI and the license). See: https://creativecommons.org/licenses/by-nc-nd/4.0/.

\section{References}

1. Magliano DJ, Islam RM, Barr ELM, et al. Trends in incidence of total or type 2 diabetes: systematic review. BMJ 2019;366:15003.

2. Katakami N. Mechanism of Development of Atherosclerosis and Cardiovascular Disease in Diabetes Mellitus. J Atheroscler Thromb 2018;25:27-39.

3. Juhan-Vage I. Haemostatic parameters and vascular risk. Atherosclerosis 1996;124 Suppl:S49-55.

4. Cortese F, Giordano P, Scicchitano P, et al. Uric acid: from a biological advantage to a potential danger. A focus on cardiovascular effects. Vascul Pharmacol 2019;120:106565.

5. Khan RMM, Chua ZJY, Tan JC, et al. From Pre-Diabetes to Diabetes: Diagnosis, Treatments and Translational Research. Medicina (Kaunas) 2019;55:546.

6. Tang Q, Li X, Song P, et al. Optimal cut-off values for the homeostasis model assessment of insulin resistance (HOMA-IR) and pre-diabetes screening: Developments in research and prospects for the future. Drug Discov Ther 2015;9:380-5.

7. Zaccardi F, Webb DR, Yates T, et al. Pathophysiology of type 1 and type 2 diabetes mellitus: a 90-year perspective. Postgrad Med J 2016;92:63-9.

8. Viigimaa M, Sachinidis A, Toumpourleka M, et al. Macrovascular Complications of Type 2 Diabetes Mellitus. Curr Vasc Pharmacol 2020;18:110-6.

9. Burggraaf $B$, Castro Cabezas M. Interventions in type 2 diabetes mellitus and cardiovascular mortality-An overview of clinical trials. Eur J Intern Med 2017;42:1-15.

10. Pareek M, Bhatt DL. Cardiometabolic risk reduction after

Cite this article as: Li L, Shi Z, Ma L, Lu Y. Analysis of the correlation between plasma coagulation factor VII, PAI1 , and uric acid with insulin resistance and macrovascular complications in elderly patients with type 2 diabetes. Ann Palliat Med 2021;10(1):664-671. doi: 10.21037/apm-20-2609 metabolic surgery. Curr Opin Cardiol 2019;34:663-72.

11. Cooper ME, Gilbert RE, Jerums G. Diabetic vascular complications. Clin Exp Pharmacol Physiol 1997;24:770-5.

12. Poredos P, Poredos P, Jezovnik MK. Structure of Atherosclerotic Plaques in Different Vascular Territories: Clinical Relevance. Curr Vasc Pharmacol 2018;16:125-9.

13. Fimognari FL, Scarlata S, Conte ME, et al. Mechanisms of atherothrombosis in chronic obstructive pulmonary disease. Int J Chron Obstruct Pulmon Dis 2008;3:89-96.

14. Gries FA, Koschinsky T. Diabetes and arterial disease. Diabet Med 1991;8 Spec No:S82-7.

15. Petersen MC, Shulman GI. Mechanisms of Insulin Action and Insulin Resistance. Physiol Rev 2018;98:2133-223.

16. Sloop GD, Weidman JJ, St Cyr JA. Atherothrombosis is a Thrombotic, not Inflammatory Disease. Cureus 2017;9:e1909.

17. Lee CJ, Sears CL, Maruthur N. Gut microbiome and its role in obesity and insulin resistance. Ann N Y Acad Sci 2020;1461:37-52.

18. Chu AJ. Tissue factor mediates inflammation. Arch Biochem Biophys 2005;440:123-32.

19. Kubala MH, DeClerck YA. The plasminogen activator inhibitor-1 paradox in cancer: a mechanistic understanding. Cancer Metastasis Rev 2019;38:483-92.

20. Yoon S, Cho H, Kim J, et al. Brain changes in overweight/ obese and normal-weight adults with type 2 diabetes mellitus. Diabetologia 2017;60:1207-17.

21. Packer M. Interplay of adenosine monophosphateactivated protein kinase/sirtuin-1 activation and sodium influx inhibition mediates the renal benefits of sodiumglucose co-transporter- 2 inhibitors in type 2 diabetes: A novel conceptual framework. Diabetes Obes Metab 2020;22:734-42.

22. Emmerson BT. Atherosclerosis and urate metabolism. Aust N Z J Med 1979;9:451-4.

(English Language Editor: A. Kassem) 